\title{
Modelling the 2-kinase domain of 6-phosphofructo-2-kinase/fructose-2,6- bisphosphatase on adenylate kinase
}

\author{
Luc BERTRAND* $\dagger$, Didier VERTOMMEN $\dagger$, Eric DEPIEREUX*, Louis HUE $\dagger$, Mark H. RIDER $\dagger \dagger$ and Ernest FEYTMANS* \\ *Facultés Universitaires Notre-Dame de la Paix, Department of Biology, Rue de Bruxelles, 61, B-5000 Namur, Belgium and †Hormone and Metabolic Research Unit, \\ University of Louvain Medical School, and International Institute of Cellular and Molecular Pathology, Avenue Hippocrate, 75, B-1200 Brussels, Belgium
}

\begin{abstract}
Simultaneous multiple alignment of available sequences of the bifunctional enzyme 6-phosphofructo-2-kinase/fructose-2,6-bisphosphatase revealed several segments of conserved residues in the 2-kinase domain. The sequence of the kinase domain was also compared with proteins of known three-dimensional structure. No similarity was found between the kinase domain of 6-phosphofructo-2-kinase and 6-phosphofructo-1-kinase. This questions the modelling of the 2-kinase domain on bacterial 6phosphofructo-1-kinase that has previously been proposed
\end{abstract}

[Bazan, Fletterick and Pilkis (1989) Proc. Natl. Acad. Sci. U.S.A. 86, 9642-9646]. However, sequence similarities were found between the 2-kinase domain and several nucleotide-binding proteins, the most similar being adenylate kinase. A structural model of the 2-kinase domain based on adenylate kinase is proposed. It accommodates all the results of site-directed mutagenesis studies carried out to date on residues in the 2kinase domain. It also allows residues potentially involved in catalysis and/or substrate binding to be predicted.

\section{INTRODUCTION}

Fructose 2,6-bisphosphate is a potent positive effector of 6phosphofructo-1-kinase (PFK-1), a key enzyme of glycolysis (for reviews see [1,2]). Its synthesis and degradation are catalysed by the bifunctional enzyme, 6-phosphofructo-2-kinase (PFK2)/fructose-2,6-bisphosphatase (FBPase-2). Several isoenzymes have been identified in eukaryotic cells. They are homodimers with two distinct catalytic sites (an N-terminal PFK-2 domain and a C-terminal FBPase-2 domain) on each subunit. The isoenzymes are termed the liver $(\mathrm{L})$, muscle $(\mathrm{M})$, heart $(\mathrm{H})$, testis (T), brain (B) and yeast (pfk26 and fbp26) isoenzymes according to their origin. They differ in molecular mass, kinetic properties and response to phosphorylation by protein kinases. The two yeast isoenzymes are monofunctional and thus differ from the others. Although they contain both domains, each yeast isoenzyme possesses only one activity, i.e. PFK-2 activity (pfk26), or FBPase-2 activity (fbp26). PFK-2/FBPase-2 isoenzyme sequences are available from a number of different species and tissues [3-13].

The three-dimensional structure of the bifunctional enzyme has not been solved (see, however, Note added in proof). On the basis of sequence alignment and modelling, Bazan et al. [14] proposed that the FBPase-2 domain is homologous with the phosphoglycerate mutase (PGM) family. All the residues in the FBPase-2 domain that have so far been studied by mutagenesis and the recent crystallographic study [15] confirmed the similarity with PGM (see [2] for a review).

Moreover, Bazan et al. [14] proposed that the PFK-2 domain was similar to bacterial 6-phosphofructo-1-kinase (PFK-1). On the basis of this modelling, several amino acid residues were proposed to play a role in catalysis and substrate binding. However, the validity of the prediction was questioned by the results of site-directed mutagenesis of several residues in this domain. These experiments showed that the positions of the active sites of the two enzymes are actually different $[16,17]$.
Indeed, in bacterial PFK-1, Asp-127 acts as a general base catalyst and Asp-129 binds $\mathrm{Mg}^{2+}$ [18-20]. According to Bazan et al. [14], these two residues correspond to Cys-160 and Asp-162 in the PFK-2 domain. Site-directed mutagenesis of Cys-160 and Asp-162 indicate that they are not involved in catalysis [16]. Moreover, Arg-72 in PFK-1 binds the $\gamma$-phosphate of ATP and stabilizes the transition state [21]. The Bazan model predicts that it corresponds to Arg-104 in the PFK-2 domain [14]. Its mutation indicates that it is involved in Fru-6-P binding and not in MgATP binding as predicted from the modelling on PFK-1 [17,22,23].

Modelling the 2-kinase domain on bacterial PFK-1 is further discredited by the fact that, according to Walker et al. [24] and Traut [25], PFK-1 possesses a unique nucleotide-binding fold (nbf), which differs from that present in most of the nucleotidebinding proteins. Different nbfs have indeed been described and can be grouped into three families: (i) the adenylate kinase $(\mathrm{AK}) / \mathrm{p} 21 \mathrm{ras} / \mathrm{EF}-\mathrm{Tu} / \mathrm{rec} \mathrm{A}$ proteins, (ii) the protein kinases and (iii) the actin/HSP-70/hexokinase group. The nbf of the AK family consists of a $\beta$-sheet surrounded by several $\alpha$-helices [24,25] and is characterized by conserved sequence patterns called Walker A and B motifs (or kinase-1 and -2 motifs) involved in binding phosphates and $\mathrm{Mg}^{2+}$ of the MgATP complex. The nbf present in PFK-1 differs from those present in the three families.

In this work, we used the multiple alignment program, Matchbox [26,27], to study the sequence and structure of the PFK-2 domain. We first identified conserved segments in the available PFK-2 domain sequences. We also studied similarities between the PFK-2 domain and other nucleotide-binding proteins and found that the nbf present in the 2-kinase domain exhibits striking similarities to that of AK. On this basis, we propose a new model for the PFK-2 domain that is consistent with all the results of mutagenesis of the residues in this domain. On the basis of this new model, several residues are predicted to play a role in substrate binding and/or catalysis. The results of

Abbreviations used: AK, adenylate kinase (EC 2.7.4.3); FBPase-2, fructose-2,6-bisphosphatase (EC 3.1.3.46); Fru-6-P, fructose 6-phosphate; nbf, nucleotide-binding fold; PFK-1, phosphofructo-1-kinase (EC 2.7.1.11); PFK-2, phosphofructo-2-kinase (EC 2.7.1.105); PGM, phosphoglycerate mutase (EC 5.4.2.1).

\$ To whom correspondence should be addressed. 
mutagenesis of several such residues are presented in the following paper [28].

\section{EXPERIMENTAL}

The PFK-2/FBPase-2 sequences included in the analysis were the L isoenzymes from rat [7], bovine [8], human [9], chicken [10] and frog [11]; the $\mathrm{M}$ isoenzymes from rat [12] and frog [11]; the rat $\mathrm{T}$ isoenzyme [3]; the rat [3] and bovine [13] $\mathrm{H}$ isoenzymes; the B isoenzyme from rat [4] and the pfk 26 and fbp26 isoenzymes from yeast [5,6]. The PFK-1 sequences were from Escherichia coli [18], Bacillus stearothermophilus [29] and human liver [30]. The PGM sequences were from yeast [31] and human muscle [32]. The sequences of AK were from yeast [33], E. coli [34], bovine heart [35] and pig muscle [36].

The Blast algorithm [37] was used for searching databases and the PHD method was used to predict secondary structures [38]. The Insight ${ }^{\mathbb{R}} I I$ (Biosym, San Diego, CA, U.S.A.) package was used to complete the structure alignment of AKs. A root mean square smaller or equal to $0.1 \mathrm{~nm}(1 \AA)$ signifies a similar structure based on $\alpha$-carbon atoms.

Sequence analyses were performed using Matchbox [26,27] on a Dec/Vax 6220 with the DFK physicochemical matrix [26], as previously described [39], and refined with the Blosum62 amino acid substitution matrix [40]. The first approach compares the observed similarities with those observed by chance between randomized sequences. Finally, boxes including similar segments were delineated. During alignments, the scanning parameter, corresponding to the shift allowed between compared segments, decreased gradually from 200 to 0 residues and the cut-off distance to match seven-residue segments was relaxed from a severe to a more tolerant matching statistical criterion as described [39].

\section{RESULTS AND DISCUSSION}

\section{Statistical analyses of the relationships between sequences}

The sequence analysis provided the distribution of the cumulated frequencies of the physicochemical differences (represented by $D$ ) between sequence segments (Figure 1). Each seven-residue segment of a sequence was compared with all seven-residue segments of the other sequences. The distribution of the cumulated frequencies of $D$ between segments obtained from the original sequences was compared with that obtained after sequence randomization. A higher frequency of similar segments in the original sequences shows a higher similarity than that expected by chance [26]. Figure 1 presents the cumulated distribution for the two least-related PFK-2/FBPase- 2 sequences, i.e. rat muscle and yeast pfk26. The difference between the distributions obtained for the original and randomized sequences indicates that they are significantly similar. As expected, this approach provides similar results for the comparison of the FBPase-2 domain with PGM. It confirms the similarity between the FBPase-2 domain and PGM and also validates our sequence analysis. In contrast, there was no difference between the distributions of $D$ for the sequence of the PFK-2 domain and bacterial PFK-1, suggesting that they are not related (Figure 1). The study was extended to test several nucleotide-binding proteins. Some of them, AK (Figure 1) and guanylate kinase (results not shown), possess some sequence similarity to the PFK-2 domain.

\section{Multiple sequence alignment of the PFK-2 domain}

To identify the conserved segments of the PFK-2 domain, we made a multiple alignment of PFK-2 sequences (Figure 2). Boxes delineate segments of highly conserved physicochemical profiles, which in principle correspond to structurally conserved regions $[26,39]$. Segments outside the boxes or separated by horizontal lines are not aligned. The regulatory domain, which is specific for each isoenzyme, is located at the $\mathrm{N}$-terminus (the first 32 amino acids of the $\mathrm{L}$ isoenzyme). Residues that have been studied by site-directed mutagenesis and shown to be important for PFK-2 activity are all found in conserved boxes (Figure 2 and Table 1). The PFK-2 domain is well conserved in all the sequences, although the number of residues totally conserved in all the sequences is low $(16 \%)$. However, about half of these residues are located in two relatively short segments (from 42 to 68 and from 118 to 142), where the percentage of identity is 30 and $50 \%$ respectively (74 and $72 \%$ similarity) corresponding to the Walker $\mathrm{A}$ and $\mathrm{B}$ motifs.

The non-conserved regions are isoenzyme-specific and may form loops or random coils located on the surface.

\section{Alignment of the PFK-2 domain with AK}

Matchbox and Blast methods were used to detect physicochemical similarities between the PFK-2 domain and other nucleotide binding proteins. The best similarities were found with $\mathrm{AK}$, the three-dimensional structure of which is known [44-46].
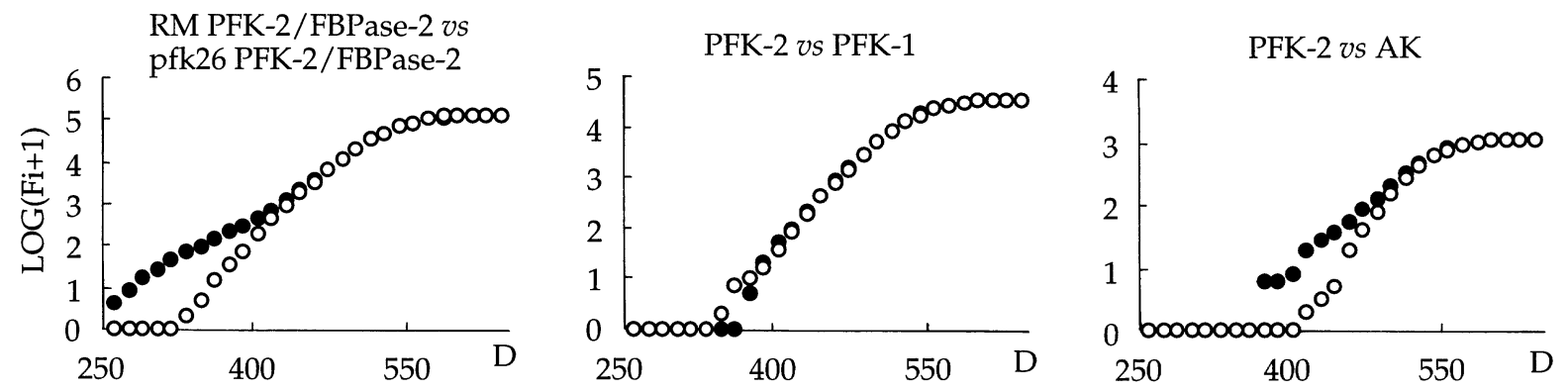

Figure 1 Comparison of matches in original sequences before $(O)$ and after $(O)$ randomization between rat muscle (RM) and pfk26 PFK-2/FBPase-2 isoenzymes, between rat liver PFK-2 and E. coli PFK-1 and between rat liver PFK-2 and E. coli AK

Fi are the cumulated frequencies of the physicochemical differences $D$ between segments of the sequences. 

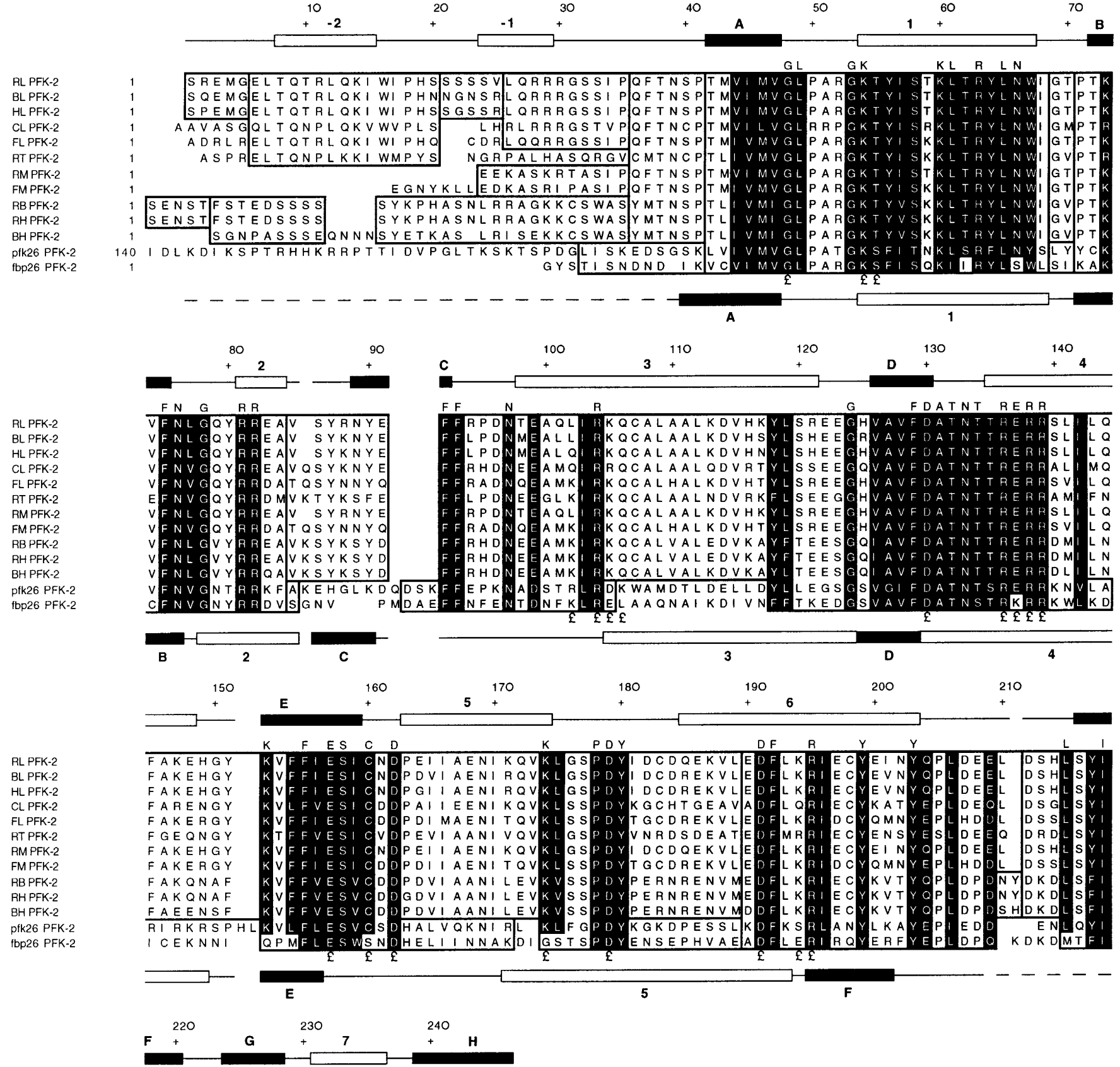

RL PFK-2

BL PFK-2

HL PFK-2

CL PFK-2

FL PFK-2

RT PFK-2

RM PFK-2

FM PFK-2

RBPFK-2

BHPFK-2

pfk26 PFK-2

fbp26 PFK-2

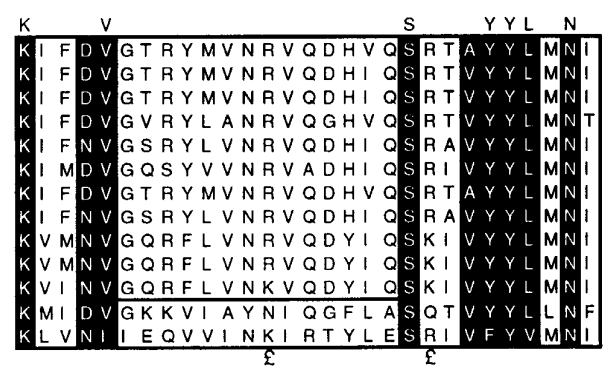

Figure 2 Multiple sequence alignment of PFK-2 sequences

The PFK-2 sequences are taken from rat liver (RL), bovine liver (BL), human liver (HL), chicken liver (CL), frog liver (FL), rat testis (RT), rat muscle (RM), frog muscle (FM), rat brain (RB), rat heart (RH), bovine heart (BH) and yeast (pfk26 and fbp26). Boxes delineate the matching regions. Letters in white on a black background show identities and similarities. Identical residues are shown above the alignment. Residues studied by site-directed mutagenesis are indicated ( $($ ) (Table 1). Predicted $\alpha$-helices (numbered in open rectangles) and $\beta$-strands (lettered in filled rectangles) made by the PHD algorithm are shown above the sequences and for the prediction of Bazan and co-workers below the sequences. 
Table 1 Amino acid residues of the PFK-2 domain studied by site-directed mutagenesis

The numbering of residues corresponds to the rat liver isoenzyme.

\begin{tabular}{lll}
\hline Residue & Suggested role & Reference \\
\hline Arg-104, Arg-225, & Fructose 6-phosphate (Fru-6-P) binding & {$[17]$} \\
Arg-138, Arg-195 & & {$[2,41]$} \\
Asp-179, Asp-191 & & {$[28]$} \\
Gly-48 & Structure of the Walker A motif & {$[2]$} \\
Lys-54 & ATP binding in the Walker A motif & {$[42]$} \\
Arg-230, Arg-238 & ATP binding & {$[2]$} \\
Asp-130 & Mg2+ binding in the MgATP complex of the Walker B & {$[43]$} \\
& motif & {$[41,42]$} \\
Thr-55, Arg-139 & Catalysis (Thr-55 is located in the Walker A motif) & {$[28]$} \\
Lys-174 & Not involved in catalysis (the corresponding residues & {$[16]$} \\
Cys-160, Asp-162 & in PFK-1, according to Bazan et al. [14] are essential & \\
& for catalysis) & \\
& Cys-160 affects affinity for MgATP & \\
& &
\end{tabular}

The multiple sequence alignment (Figure 3 ) shows that several residues in the Walker $\mathrm{A}$ and $\mathrm{B}$ motifs involved in MgATP binding and in stabilization of the transition state are present in the two enzymes. They correspond to the sequences that are conserved in the 2-kinase domain (Figure 2).

(i) The secondary structure of the first region is predicted to be composed of a $\beta$-strand/loop/ $\alpha$-helix structure. It contains a sequence found in nucleotide-binding proteins $\left(\mathrm{G}-48 \mathrm{X}_{4} \mathrm{GKT}\right.$ 55), which corresponds to the Walker A motif [24,25]. The $\alpha$ helix following the glycine-containing loop contains in PFK-2 several conserved residues (Figure 2): Lys-54, Thr-55, Ser-58, Lys-60, Thr-62 and Arg-63. Mutation of the conserved Lys residue to Met in both AK and PFK-2 (Lys-54) decreased the $k_{\text {cat }}$ by three orders of magnitude [42,47]. The crystal structure of AK suggests that this lysine binds the $\beta$ - and $\gamma$-phosphates of MgATP. The adjacent residue, Thr-55, is conserved in all the PFK-2 sequences. A similar residue is found in the same family of nucleotide-binding enzymes: AK (Thr-15), p21 Ha-ras (Ser-17), rec A (Thr-73), EF-Tu (Thr-25). X-ray crystallography of these enzymes suggests that this $\mathrm{Thr} / \mathrm{Ser}$ binds $\mathrm{Mg}^{2+}$ in the MgATP complex [44-46,48,49]. Mutagenesis of Thr-55 to Val, Cys or Ser in the PFK-2 domain indicates that Thr-55 is important for catalysis (Table 1). Moreover, mutation of Gly-48, the first glycine residue in the loop between the $\beta$-sheet and $\alpha$-helix, abolishes PFK-2 activity (Table 1). However, it is not known whether this glycine residue plays a structural role in the nbf.

(ii) The second segment is predicted to be composed of the Walker B motif (end of $\alpha 3$-helix/loop/ $\beta$ D-strand) followed by a
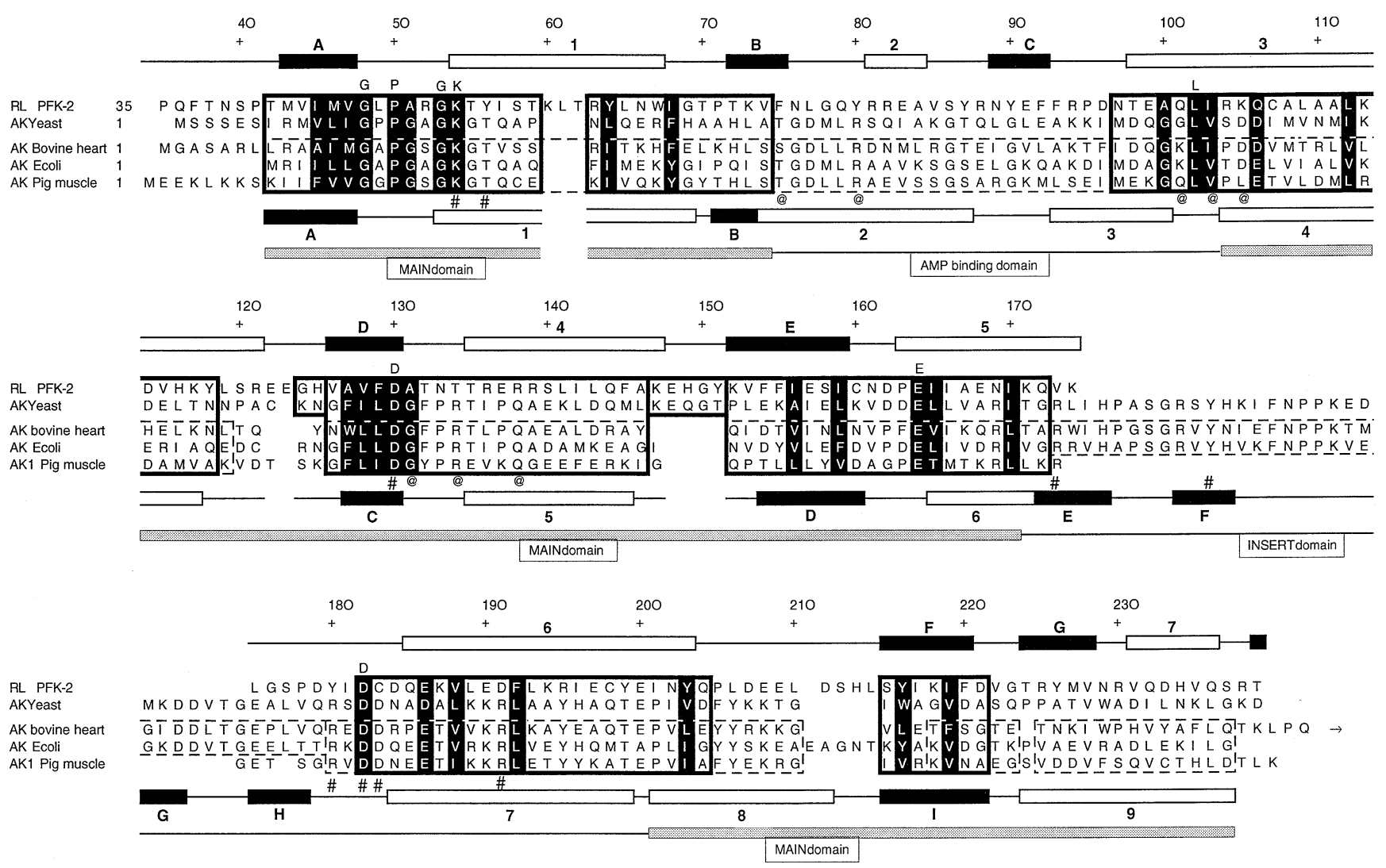

Figure 3 Multiple sequence alignment of the PFK-2 domain with AKs

Solid boxes delineate matching regions between the sequences (only boxes including the PFK-2 domain are shown). Dashed boxes represent structurally conserved regions between AK structures. Letters in white on a black background show identities and similarities. Identical residues are shown above the alignment. Residues that bind MgATP (\#) and AMP (@) in the AKs are indicated. Predicted $\alpha$-helices (open rectangles) and $\beta$-strands (filled rectangles) for the PFK-2 domain are shown above the sequences. $\alpha$-Helices (open rectangles) and $\beta$-strands (filled rectangles) from the three-dimensional structure of $E$. coli AK are shown below the sequences. Secondary structures are numbered according to $E$. coli AK. 
A

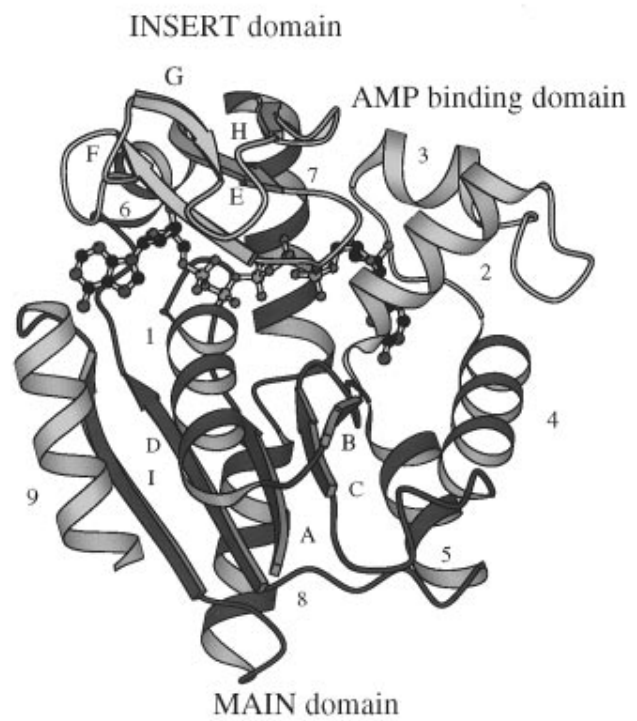

B

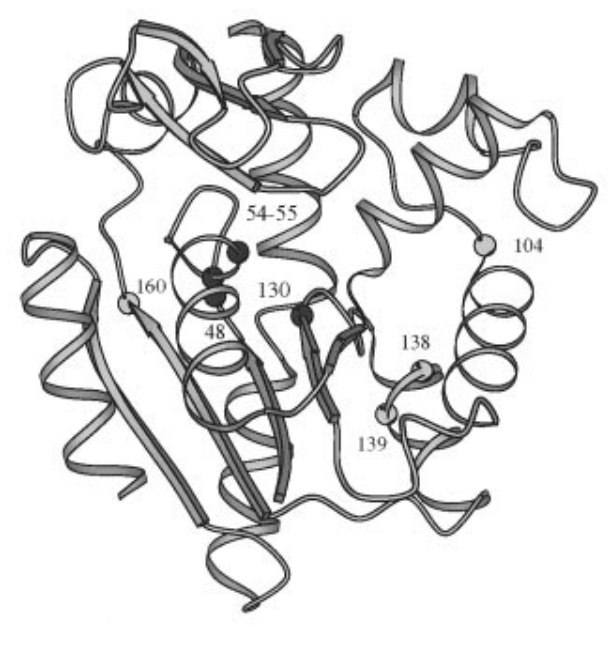

Figure 4 Molscript [50] representation of the three-dimensional structure of AK from $E$. coli complexed with the inhibitor $\mathrm{P}^{1}, \mathrm{P}^{5}$-diadenosine 5'-pentaphosphate

The MgATP-binding site is located on the left and the AMP-binding site is on the right. The predicted regions between AKs and PFK-2 which show similarity are represented in black (A). Secondary structures are numbered according to $E$. coli AK. The numbering of residues corresponds to the rat liver PFK-2 isoenzyme. In conserved regions, residues playing the same role in the two enzymes are represented in black. Residues specific to PFK-2 are shown in white (B).

loop/ $\alpha 4$-helix structure. This region is particularly well conserved. It contains several conserved residues of PFK-2 (Figure 2): Asp-130, Thr-132, Asn-133, Thr-134, Thr-135, Arg-136, Glu137, Arg-138, Arg-139. Some of these have recently been studied by site-directed mutagenesis in our laboratory (Table 1). Asp-130 binds $\mathrm{Mg}^{2+}$ of the MgATP complex and is important for catalysis, Arg-138 binds Fru-6-P and Arg-139 probably participates in catalysis by stabilizing the transition state.

The three-dimensional structure of the MgATP-binding domain of AK shows a topology that is similar to the predicted secondary structure of the PFK-2 domain (Figures 4 and 5). AK is composed of three domains. The so-called 'MAIN' domain comprises the protein core and is formed by a five-strand parallel $\beta$-sheet ( $\beta \mathrm{I}-\beta \mathrm{D}-\beta \mathrm{A}-\beta \mathrm{C}-\beta \mathrm{B})$ surrounded by $\alpha$-helices $(\alpha 9-\alpha 1-\alpha 8$ $\alpha 5)$. Except for the $\alpha 9$-helix, Matchbox and the PHD method show that the entire structure of the MAIN domain of AK can be found in the PFK-2 domain. The two other domains of AK are not conserved in the PFK-2 domain. One is involved in AMP binding ( $\alpha 2-\alpha 3)$. The other, called the 'INSERT' domain, is composed of a four-strand antiparallel $\beta$-sheet $(\beta \mathrm{E}-\beta \mathrm{F}-\beta \mathrm{G}-\beta \mathrm{H})$. It is involved in the induced-fit movement driving catalysis and contains residues involved in stabilization of the transition state during in-line phosphate transfer. The structure of AK has been used to model the three-dimensional structure of the PFK-2 domain (Figure 5).

\section{Position of mutated residues in the new model of the PFK-2 domain}

Modelling the 2-kinase domain on AK accommodates all previous mutagenesis studies (Table 1).

\section{MgATP-binding domain}

In the model, four regions are located in the $\mathrm{MgATP}$-binding domain. The first and second are the Walker A and Walker B motifs and have already been discussed (see above). The third region (loop between the $\beta$ E-strand and $\alpha 5$-helix) is situated between the MAIN domain and the INSERT domain. Cys-160 is located in this loop where it could interact with the ribose or adenine rings of MgATP. This may explain why Cys-160 can be labelled by iodoacetamide in the absence of substrates, but not in the presence of MgATP, and by the nucleotide analogue fluorosulphonylbenzoyladenosine [51]. Moreover, mutation of Cys-160 to aspartate increased the $K_{\mathrm{m}}$ for MgATP 15-fold [16].

The fourth region is the $\beta \mathrm{G}$-strand and $\alpha 7$-helix. This region is not present in $\mathrm{AK}$ and possesses an unknown tertiary structure. The $\alpha 7$-helix is flanked by two residues, Arg-230 and Arg-238, which have been studied by site-directed mutagenesis and shown to be involved in MgATP binding (Table 1).

\section{Fru-6-P-binding domain}

The AMP-binding domain in AK is not conserved in the PFK2 domain. In PFK-2, this region is probably involved in Fru-6$\mathrm{P}$ binding. It seems to be delimited by the $\beta \mathrm{B}$-strand and $\alpha 3$-helix (Figure 5, residues 75 to 104) and contains several residues conserved in all PFK-2 sequences: Asn-76, Arg-81, Arg-82, Asn97, Glu-99 and Arg-104. Site-directed mutagenesis of Arg-104 indicated that this residue is indeed involved in Fru-6-P binding (Table 1).

The region of the PFK-2 domain corresponding to the INSERT domain of AK contains several residues conserved in all PFK-2 sequences. Arg-195 has been studied and binds Fru-6$\mathrm{P}$ (Table 1). The region contains two conserved aspartate residues 

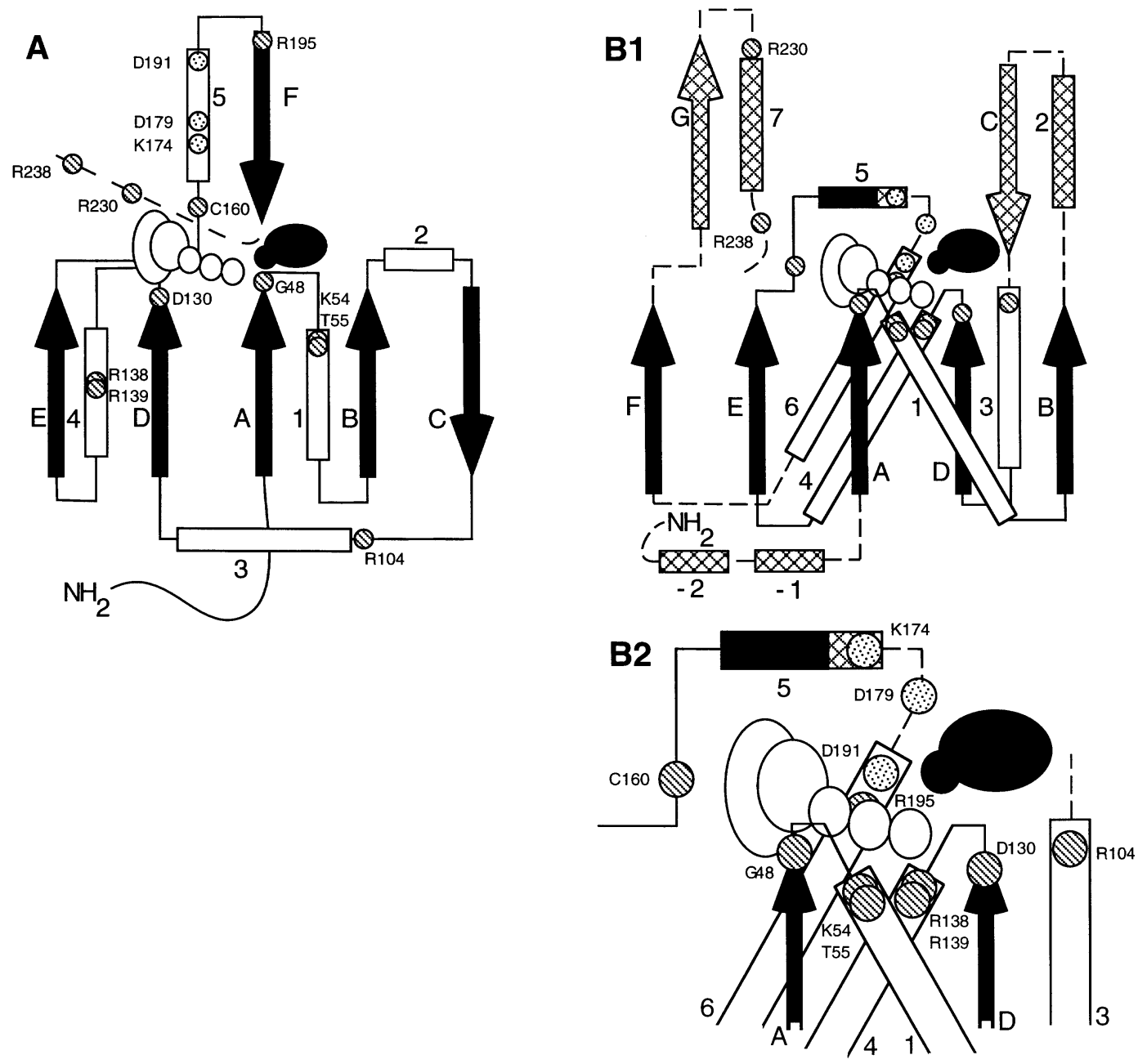

Figure 5 Comparison of the topology of the two PFK-2 domain models based on the analogy with bacterial PFK-1 (A) and the similarity with AK (B)

The $\beta$-strands and $\alpha$-helices are shown as arrows and boxes respectively. They are numbered as in Figure 3. MgATP and Fru-6-P are represented by unfilled and filled ovals respectively. Residues involved in catalysis or in substrate binding are represented by hatched and dotted (for residues studied in the following paper [28]) circles. Cross-hatched secondary structures in global view (B1) and in an enlarged view of the active site (B2) are specific to the PFK-2 domain. The other secondary structures are common to PFK-2 and AK.

(Asp-179 and Asp-191) and a conserved lysine residue (Lys-174, replaced by a glycine in the fbp26 yeast isoenzyme). Site-directed mutagenesis of these three residues has been studied, and the results presented in the following paper [28] confirm their role in substrate binding and catalysis.

\section{Conclusion}

The three-dimensional structure of the PFK-2 domain has been suggested to resemble that of bacterial PFK-1 [14]. On the contrary, our sequence analyses show that the PFK-1 and PFK2 domains are unrelated. This confirms the negative site-directed mutagenesis studies on residues in the PFK-2 that were proposed to be important for catalysis [16].

Our analysis shows that the predicted structure of the PFK-2 domain corresponds to the topology of AK. Figure 5 compares the connectivity of the secondary structure of PFK-2, based on the analogy with bacterial PFK-1, with that described here. The model and alignment of all available PFK-2 sequences implicate several residues to be involved in substrate binding or catalysis. Some of these have been tested by site-directed mutagenesis
(Table 1) and confirm the validity of the theoretical study. Moreover, we can speculate that several other residues would be involved in catalysis or substrate binding. These are Asn-76, Arg81, Arg-82, Asn-97, Glu-99, Thr-132, Asn-133, Thr-134, Thr135. All these residues are conserved in the PFK-2 domain and are located near the catalytic and substrate-binding sites. Therefore the model proposed can accommodate all the site-directed mutagenesis studies obtained so far and could help in targeting new residues essential for catalysis and substrate binding.

\section{Note added in proof (received 29 November 1996)}

The three-dimensional structure of PFK-2/FBPase-2 was solved and published [52] after completion of our manuscript. It entirely confirms the structure model of the PFK-2 domain, based on adenylate kinase, presented in this paper.

We thank G. G. Rousseau for continued interest and Y. Marchand for secretarial assistance. L.B. and D.V. were supported by grants from the IRSIA (FRIA), Belgium. M.H.R. is a Research Associate of the National Fund for Scientific Research (Belgium). This work was supported by the D.G. Higher Education and Scientific 
Research - French Community of Belgium, by the Caisse Générale d'Epargne et de Retraite, and by the Fund for Medical Scientific Research (Belgium).

\section{REFERENCES}

1 Hue, L. and Rider, M. H. (1987) Biochem. J. 245, 313-324

2 Pilkis, S. J., Claus, T. H., Kurland, I. J. and Lange, A. J. (1995) Annu. Rev. Biochem. 64, 799-835

3 Sakata, J., Abe, Y. and Uyeda, K. (1991) J. Biol. Chem. 266, 15764-15770

4 Watanabe, F., Sakai, A., Furuya, E. and Uyeda, K. (1994) Biochem. Biophys. Res. Commun. 198, 335-340

5 Kretschmer, M. and Fraenkel, D. G. (1991) Biochemistry 30, 10663-10672

6 Paravicini, G. and Kretschmer, M. (1992) Biochemistry 31, 7126-7133

7 Darville, M. I., Crepin, K. M., Vandekerckhove, J., Van-Damme, J., Octave, J. N., Rider, M. H., Marchand, M. J., Hue, L. and Rousseau, G. G. (1987) FEBS Lett. 224, 317-321

8 Lange, A. J., El-Maghrabi, M. R. and Pilkis, S. J. (1991) Arch. Biochem. Biophys. 290, 258-263

9 Algaier, J. and Uyeda, K. (1988) Biochem. Biophys. Res. Commun. 153, 328-333

10 Li, L., Lange, A. J. and Pilkis, S. J. (1993) Biochem. Biophys. Res. Commun. 190, 397-405

11 Sakai, A., Watanabe, F. and Furuya, E. (1994) Biochem. Biophys. Res. Commun. 198, 1099-1106

12 Crepin, K. M., Darville, M. I., Hue, L. and Rousseau, G. G. (1989) Eur. J. Biochem. 183, 433-440

13 Sakata, J. and Uyeda, K. (1990) Proc. Natl. Acad. Sci. U.S.A. 87, 4951-4955

14 Bazan, J. F., Fletterick, R. J. and Pilkis, S. J. (1989) Proc. Natl. Acad. Sci. U.S.A. 86, 9642-9646

15 Lee, Y.-H., Ogata, C., Pflugrath, J. W., Levitt, D. G., Sarma, R., Banaszak, L. J. and Pilkis, S. J. (1996) Biochemistry 35, 6010-6019

16 Crepin, K. M., Vertommen, D., Dom, G., Hue, L. and Rider, M. H. (1993) J. Biol. Chem. 268, 15277-15284

17 Rider, M. H., Crepin, K. M., De Cloedt, M., Bertrand, L., Vertommen, D. and Hue, L. (1995) Biochem. J. 309, 341-346

18 Hellinga, H. W. and Evans, P. R. (1987) Nature (London) 327, 437-439

19 Laine, R., Deville-Bonne, D., Auzat, I. and Garel, J.-R. (1992) Eur. J. Biochem. 207, 1109-1114

20 Berger, S. A. and Evans, P. R. (1992) Biochemistry 31, 9237-9242

21 Berger, S. A. and Evans, P. R. (1990) Nature (London) 343, 575-576

22 Kurland, I., Chapman, B., Lee, Y.-H. and Pilkis, S. J. (1995) Biochem. Biophys. Res. Commun. 213, 663-672

23 Tsujikawa, T., Watanabe, F. and Uyeda, K. (1995) Biochemistry 34, 6389-6393

24 Walker, J. E., Saraste, M., Runswick, M. J. and Gay, N. J. (1982) EMBO J. 1, 945-951
25 Traut, T. W. (1994) Eur. J. Biochem. 222, 9-19

26 Depiereux, E. and Feytmans, E. (1991) Protein Eng. 4, 603-613

27 Depiereux, E. and Feytmans, E. (1992) Comput. Appl. Biosci. 8, 501-509

28 Bertrand, L., Deprez, J., Vertommen, D., Di Pietro, A., Hue, L. and Rider, M. H. (1997) Biochem. J. 321, 623-627

29 French, B. A. and Chang, S. H. (1987) Gene 54, 65-71

30 Elson, A., Levanon, D., Brandeis, M., Dafni, N., Bernstein, Y., Danciger, E. and Groner, Y. (1990) Genomics 7, 47-56

31 White, M. F. and Fothergill-Gilmore, L. A. (1988) FEBS Lett. 229, 383-387

32 Castella-Escola, J., Ojcius, D. M., Le Boulch, P., Joulin, V., Blouquit, Y., Garel, M. C., Valentin, C., Rosa, R., Climent-Romeo, F. and Cohen-Solal, M. (1990) Gene 91 225-232

33 Magdolen, V., Oechsner, U. and Bandlow, W. (1987) Curr. Genet. 12, 405-411

34 Brune, M., Schumann, R. and Wittinghofer, F. (1985) Nucleic Acid Res. 13, 7139-7151

35 Yamada, M., Shahjahan, M., Tanabe, T., Kishi, F. and Nakazawa, A. (1989) J. Biol. Chem. 264, 19192-19199

36 Heil, A., Muller, G., Noda, L., Pinder, T., Schirmer, H., Schirmer, I. and von Zabern, I. (1974) Eur. J. Biochem. 43, 131-144

37 Altschul, S. F., Gish, W., Miller, W., Myers, E. W. and Lipman, D. J. (1990) J. Mol. Biol. 215, 403-410

38 Rost, B., Sander, C. and Schneider, R. (1994) Comput. Appl. Biosci. 10, 53-60

39 Vinals, C., Depiereux, E. and Feytmans, E. (1993) Biochem. Biophys. Res. Commun. 192, $182-188$

40 Henikoff, S. and Henikoff, J. G. (1992) Proc. Natl. Acad. Sci. U.S.A. 89 10915-10919

41 Bertrand, L., Vertommen, D., Feytmans, E., Di Pietro, A., Rider, M. H. and Hue, L. (1997) Biochem. J. 321, 609-614

42 Vertommen, D., Bertrand, L., Sontag, B., Di Pietro, A., Louckx, M. P., Vidal, H., Hue, L. and Rider, M. H. (1996) J. Biol. Chem. 271, 17875-17880

43 Rider, M. H., Crepin, K. M., De Cloedt, M., Bertrand, L. and Hue, L. (1994) Biochem. J. 300, 111-115

44 Schulz, G. E., Elzinga, M., Marx, F. and Schrimer, R. H. (1974) Nature (London) 250 120-123

45 Diederichs, K. and Schulz, G. E. (1991) J. Mol. Biol. 217, 541-549

46 Muller, C. W. and Schulz, G. E. (1992) J. Mol. Biol. 224, 159-177

47 Byeon, L., Shi, Z. and Tsai, M. D. (1995) Biochemistry 34, 3172-3182

48 Kjeldgaard, M. and Nyborg, J. (1992) J. Mol. Biol. 223, 721-742

49 Logan, K. M. and Knight, K. L. (1993) J. Mol. Biol. 232, 1048-1059

50 Kraulis, P. J. (1991) J. Appl. Crystallogr. 24, 946-950

51 El-Maghrabi, M. R., Pate, T. M., D’Angelo, G., Correia, J. J., Lively, M. 0. and Pilkis, S. J. (1987) J. Biol. Chem. 262, 11714-11720

52 Hasemann, C. A., Istvan, E. S., Uyeda, K. and Deisenhofer, J. (1996) Structure 4 $1017-1029$

Received 16 July 1996/26 September 1996; accepted 26 September 1996 